ARTICLE

Check for updates

https://doi.org/10.1038/s41467-020-15288-8

OPEN

\title{
A flexible topo-optical sensing technology with ultra-high contrast
}

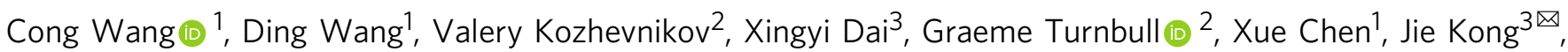
Ben Zhong Tang (i) ${ }^{4 凶}$, Yifan Li (i) ${ }^{1 凶} \&$ Ben Bin Xu (i) ${ }^{1 凶}$

Elastic folding, a phenomenon widely existing in nature, has attracted great interests to understand the math and physical science behind the topological transition on surface, thus can be used to create frontier engineering solutions. Here, we propose a topo-optical sensing strategy with ultra-high contrast by programming surface folds on targeted area with a thin optical indicator layer. A robust and precise signal generation can be achieved under mechanical compressive strains $(>0.4)$. This approach bridges the gap in current mechanoresponsive luminescence mechanism, by utilizing the unwanted oxygen quenching effect of Iridium-III (Ir-III) fluorophores to enable an ultra-high contrast signal. Moreover, this technology hosts a rich set of attractive features such as high strain sensing, encoded logic function, direct visualisation and good adaptivity to the local curvature, from which we hope it will enable new opportunities for designing next generation flexible/wearable devices.

\footnotetext{
${ }^{1}$ Department of Mechanical and Construction Engineering, Faculty of Engineering and Environment, Northumbria University, Newcastle upon Tyne NE1 8ST, UK

${ }^{2}$ Department of Applied Sciences, Faculty of Health and Life Science, Northumbria University, Newcastle upon Tyne NE1 8ST, UK. ${ }^{3}$ MOE Key Laboratory of Material Physics and Chemistry under Extraordinary, Shaanxi Key Laboratory of Macromolecular Science and Technology, School of Chemistry and Chemical Engineering, Northwestern Polytechnical University, Xi'an 710072, China. ${ }^{4}$ Department of Chemistry, The Hong Kong Branch of Chinese National Engineering Research Center for Tissue Restoration and Reconstruction and Institute for Advanced Study, The Hong Kong University of Science and Technology, Clear

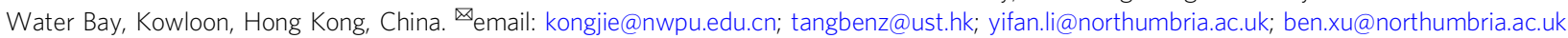


O ne of the latest trends in next generation micro-electronics technology is to develop flexible optical sensors and actuators, which holds promises in strain/pressure sensing $^{1-4}$, wearable devices ${ }^{5-7}$, electronic skin $^{8-10}$, camouflaging ${ }^{11}$, etc. By utilizing soft materials, recent efforts have explored the flexible optical technology with extra controllability and ondemand color changing such as triboelectric-photonic ${ }^{12,13}$, piezoelectroluminescent ${ }^{14}$, piezo-photonics ${ }^{15-17}$, mechano-responsive luminescence (MRL) and mechanochromism ${ }^{18}$. Among those approaches, MRL, a tunable and switchable luminescence (or chromism) in response to mechanical stimulus ${ }^{19,20}$, have attracted considerable interests for their potentials in sensing/micro-devi$\mathrm{ces}^{21}$, data storage $\mathrm{e}^{22,23}$, flexible display ${ }^{24,25}$, security pattern/ inks ${ }^{26}$, etc. However, the optical performance has been discounted by aggregation-caused quenching (ACQ) ${ }^{27,28}$, thus limit the further applications for MRL materials. Whereas the current advances in Aggregation-induced emission (AIE) have achieved emergence characteristics at molecular level to overcome the drawbacks of $\mathrm{ACQ}^{29-31}$, novel optical sensing mechanisms remain yet to be exploited to enable wider scale-up perspectives.

Inspired by epidermal color changing scheme from nature, researchers have been able to amplify signal by generating luminescent molecular dominos ${ }^{19}$ thus realize multi-state optical switching by engineering micro/nano-structures on surface ${ }^{18}$. By far, all practiced strategies will easily result into a noisy and low-resolution signal, which poses challenges in triggering controllable signals for scalable applications. Subject to the mechanical stimuli, elastomeric materials can undergo surface morphological change (e.g. wrinkles and cracks) which has been used to create switchable optical features ${ }^{25,32}$, and structural colour with dynamic luminescent patterns ${ }^{33}$. Zeng et al reported an interesting mechanochromic device by using cracks and folds ${ }^{34}$ to trigger optical signals within a moderate stretching strain of 0.2 . While the understandings on controllably generating elastic instability morphologies have been extended ${ }^{35-38}$, even to form 3D structures ${ }^{39-41}$, surface topology enabled optical sensing in response to large compressive strain (more than 0.4) has not been reported elsewhere.

In this work, we propose a topo-optical sensing strategy with ultra-high contrast by constructing a patterned elastic multilayer coated with a nanometer thin optical indicator layer. The keys to achieve such high contrast topo-sensing strategy include the targeted folding on elastic surface guided by the pre-defined lattice pattern and the autonomous optimization of contrast by selectively oxygen quenching of the coated Iridium-III complexes (Ir-III) fluorophore layer. The unique self-contact geometry of folding area preserves intensity by mechanically creating a hypoxia zone, whereas the intensity reduces significantly for the rest of surface due to the oxygen-quenching at the open air. Moreover, we successfully demonstrate several conceptual designs based on this topo-sensing approach such as an in-plane strain sensor, a $2 \mathrm{D}$ spy barcode, an adaptive topo-optical grid with potential for bio-applications and a flexible bending sensor, to shed the lights on the future applications in micro-devices and flexible/wearable electronics.

\section{Results}

Configuration of targeted folding on elastic multilayer. The multilayer system consists of a soft polydimethylsiloxane film (PDMS, shear modulus $G_{\text {sub }} \approx 0.15 \mathrm{MPa}$, thickness of $125 \mu \mathrm{m}$ ) on a vinylpolysiloxane mounting substrate (VPS, shear modulus $\approx$ $0.35 \mathrm{MPa}$, thickness of $1.5 \mathrm{~mm}$ ). Oxygen plasma was applied to create a hard skin layer (shear modulus $G_{\mathrm{f}} \approx 1.8 \mathrm{MPa}$, thickness of $100 \mathrm{~nm}$ ) on the top of PDMS film. By applying a uniaxial compression (Fig. 1a), $\varepsilon_{\text {comp }}=\frac{L_{0}}{L}-1$, an elastic morphological development is shown on the surface. A similar setting has been previously used to configure wrinkle pattern by pre-placing Bravais lattice holes on the surface at low compression ${ }^{42}$, where an unexpected formation of wrinkle-to-crease/folding transition was discovered occasionally under a higher compression $(\varepsilon>0.4)$ but have not been studied further. The key in this work is to investigate the controllable formation of targeted crease/fold at higher compression and translate this topographical transition into a dedicated sensing signal in responding to a compressive strain.

We first compare the development of elastic morphologies with reflective optical microscopy between a plain (Fig. 1b) and a patterned surface with a single-line array of micro-holes as shown in Fig. 1c (diameter $=60 \mu \mathrm{m}$, distance $D=120 \mu \mathrm{m}$, hole depth $h$ $=12 \mu \mathrm{m}$, Supplementary Movie 1). Wrinkle patterns are developed globally for both plain and centre lattice hole patterned surfaces at low compressive strains and evolved into visible textures when strain increases to $\varepsilon=0.27$. A strain energy localization guided by the pre-placed pattern can be clearly identified along the micro-holes array. The surface presents a post-wrinkling development with mixed morphologies at middle compressive strains (i.e. $\varepsilon=0.38$ ). According to Kim and coworkers, surface wrinkles will first undergo period doubling, followed by the formation of creases under a modulus ratio $\left(G_{\mathrm{f}} / G_{\text {sub }}\right)$ between 5.86 and $13.89^{43,44}$. A threshold strain $\left(\varepsilon_{\text {th }}\right)$ is the compressive strain when the first fold occurs on surface, which is variable against the setting factors for multilayer. Here, a compressive strain of $\varepsilon=0.52$, which is slightly higher than $\varepsilon_{\mathrm{th}}$, is chosen to compare folding conditions at the same energy level. We find that a few random folds (pointed by red arrows in Fig. 1b) appear on the plain surface, while a single big fold locates at the area that is defined by the pre-placed holes on surface (dotted line in Fig. 1c). Single-line array with varied pattern shapes (circles, diamonds, squares, triangles and hexagons, Supplementary Fig. 1) and different $D / \Phi$ (Supplementary Fig. 2) are also attempted, where a range of $\varepsilon_{\text {th }}$ from 0.42 to 0.58 can be achieved by designing the shape and $D / \Phi$. However, the $\varepsilon_{\text {th }}$ shows less sensitivity on the depth of lattice pattern (Supplementary Fig. 3), which agrees with the reported results on configuring the wrinkle patterns with Bravais lattice ${ }^{45}$.

Realization of topo-optical sensing. The concept of translating surface topology into optical signal (Topo-optical sensing) is initially facilitated (Fig. 1a) through casting and drying a drop of solution containing $1.3 \mathrm{mM}$ fluorescein o-acrylate (FoA) on the elastic surface, to fulfil the photo-luminescence function. When the fold occurs, the in-plane length $(L)$ locally develops into a selfcontact depth $\left(H_{c}\right.$, Fig. 1a), lead to an optical signal from the top view because of the volumetric accumulation of intensity. By assessing the optical properties for the morphologies at $(\varepsilon=0.52)$ for both plain and micropatterned surfaces under laser scanning confocal microscopy (LSCM, Fig. 1d, e), a single-line optical signal is clearly shown on the location defined by the micropattern with an enhanced intensity (side view). We define a signal-to-noise ratio (SNR) as, SNR $=\frac{\text { peak intensity }}{\text { noise }}$, to quantitatively analyse the optical signal, where the peak intensity is collected from the signal of folded line and the noise represent the average luminescence signals originated from the background surface (excluding the folding line). In Fig. 1f, a higher SNR value is obtained for the patterned surface than that of plain surface. With a hole array, surface energy can be guided to form a single fold with a deeper self-contact (Fig. 1e), rather than a distractive energy localization with multiple folds/creases on the plain surface. From the LSCM 3D reconstruction image (green opened book, Fig. 1g) for the FoA patterned surface, we note that the 
a

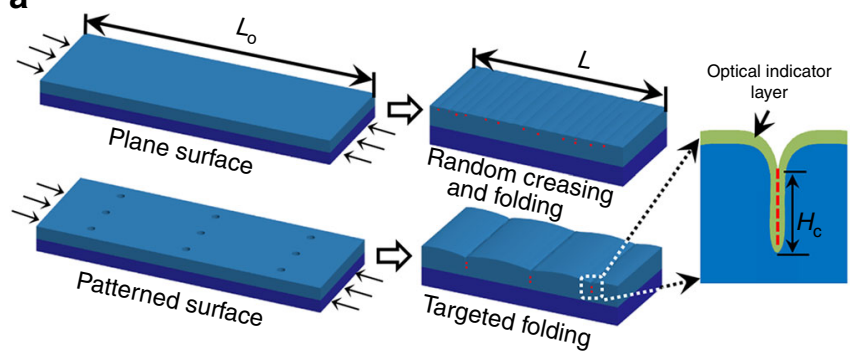

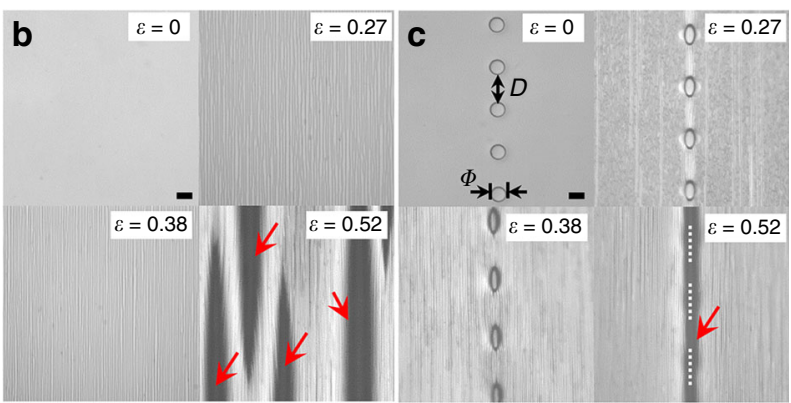

f

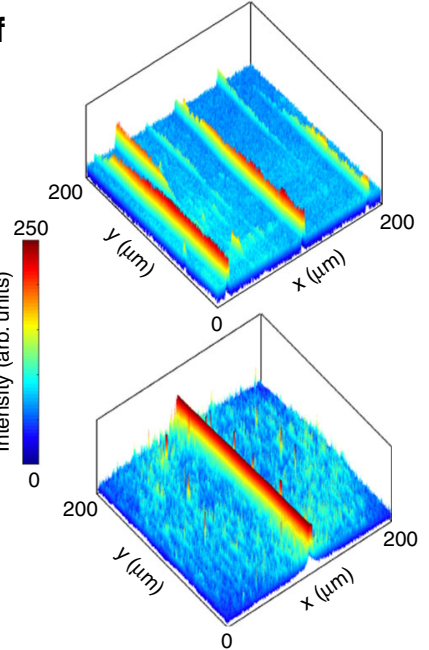

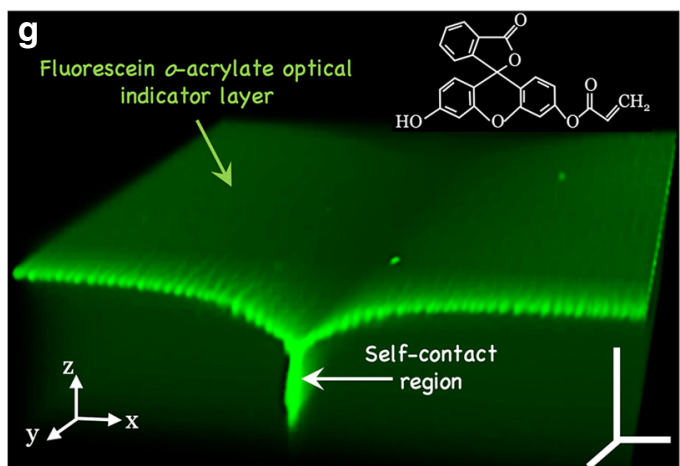

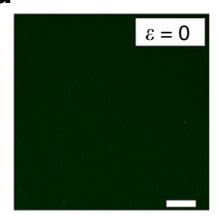

e

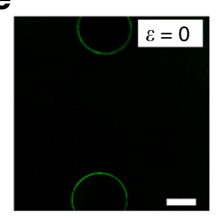

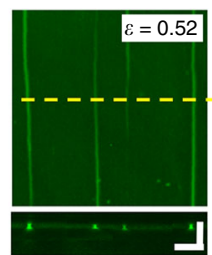

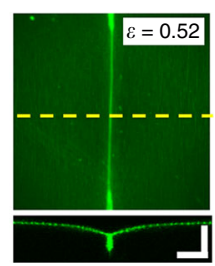

Fig. 1 The realization of topo-optical sensing. a Schematic illustrations and optical microscopic observations on the morphological changes on (b) plain surface and (c) surface patterned with single-line of micro-hole array $(\Phi=60 \mu \mathrm{m}, D=120 \mu \mathrm{m}, h=12 \mu \mathrm{m}$, dot lines showing the location of closed holes) under compression. Laser scanning confocal microscopy (LSCM) images showing top and cross-sectional (for the dash lines in top view images) views of signals induced by (d) random folding on the plain surface, and (e) targeted folding on a micropatterned surface, with the digital analysed results for top view optical signals in (f). $\mathbf{g}$ A signal image of green opened book from 3D LSCM reconstruction to show the surface signal with targeted folding. All scale bars are $30 \mu \mathrm{m}$.

signal noise on background remains considerably high, due to the discontinuous fluorescein aggregation caused by small creases/ folds ${ }^{45}$.

Selectively oxygen-quenching induced ultra-high contrast. The Iridium-III (Ir-III) complex is an oxygen-quenching phosphorescent material which usually emits orange-red coloured light $\left(\lambda_{\text {emission }}=580 \mathrm{~nm}\right.$, Fig. $\left.2 \mathrm{a}\right)$ in hypoxia condition after being excited ${ }^{45}$. Our aim is to utilize the topological hypoxia zone, created by targeted folding, to preserve the optical signal on the self-contact region for the Ir-III coating layer (Fig. 2b). Exposed to the oxygen in open air, Ir-III complex luminescence outside of the folding area is mostly eliminated by the oxygen quenching effect, leading to a topo-optical signal with ultrahigh contrast (see "blade" pattern in Fig. 2c and intensity analysis in Fig. 2d). A nominal line contrast (NLC) is defined as $\mathrm{NLC}=\frac{\text { peak intensity }}{\text { mean average of theline intensity }}$, to describe the optical signal distribution for the selected area. After analysing the NLC data (Fig. 2e) for the selected lines in Figs. 2b, 1e, an NLC value of 10 is achieved for the Ir-III coated surface, which is 5 folds of the NLC ( 2) for FoA coated surface.

We next scale this topo-optical relationship (SNR versus $H_{c}$, Fig. $2 \mathrm{f}$ ) to understand the geometrical influence on the quality of signal. Small SNRs of $0.47 \pm 0.04$ are captured when the fold first occurs at $\varepsilon_{\text {th }}$ with an onsite $H_{\mathrm{c}} \approx 1.1 \mu \mathrm{m}$, for both Ir-III and FoA coated surfaces. When $H_{\mathrm{c}}$ grows higher than $1.6 \mu \mathrm{m}$, a stable SNR plateau $(\mathrm{SNR} \geq 2)$ is emerging for FoA coated surface which indicate that the physical accumulation along the folding depth reaches a threshold of intensity to enable a quality optical feedback. This development of self-contact depth, around $500 \mathrm{~nm}$ in distance, is very rapid within a strain window of $\sim 0.037 \pm$ 0.017 . Given by a nominal strain speed of $0.02 \mathrm{~s}^{-1}$, the sensing signal can be instantly captured in microscope within $1 \mathrm{~s}$. In contrast, an increasing trend is obtained for Ir-III coated surface at the same threshold when $H_{c}$ increases, due to the oxygen quenching effect at the open air. When the $H_{c}$ reaches $13.8 \mu \mathrm{m}$, the SNR on Ir-III coated surface increases significantly to 12.5 which is 6 folds of that from FoA coated surface. By preserving the peak intensity at self-contact area, the Ir-III coated surface achieve higher SNRs when $H_{c}$ is larger than $1.6 \mu \mathrm{m}$.

The time-dependent degradation of photoluminescent signal is assessed by tracking the peak and background signals at $\varepsilon_{\text {comp }}=$ $0.55\left(H_{\mathrm{c}} \sim 13.8 \mu \mathrm{m}\right)$ for up to $200 \mathrm{~h}$. The results for FoA coated surface (Fig. $2 \mathrm{~g}$ ) show a retainment of intensity after $200 \mathrm{~h}$ with less than $8 \%$ decay for both peak and background signals. For IrIII coated surface, a rapid decrease of around $92 \%$ intensity is discovered in first $6 \mathrm{~h}$ for the background signal, whilst the peak signal remains stable for the first $70 \mathrm{~h}$, then starts to fade and reaches a decrease of $54 \%$ in $200 \mathrm{~h}$. The quenching kinetics is analysed for the Ir-III films on PDMS surfaces with varied thicknesses (Supplementary Fig. 4) in the open air, where the optical signals are quenched for about $5 \mathrm{~h}$ in all layers without compression. Under compression, the topology enabled hysteresis results (Fig. 2g) into a high optical contrast for about $65 \mathrm{~h}$, then the peak intensity eventually reduces due to the diffusion of 
a

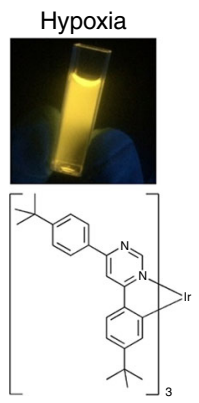

b

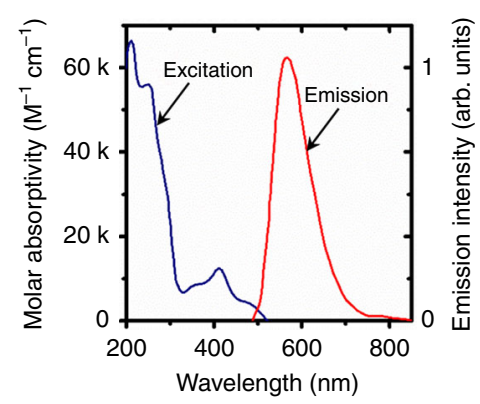

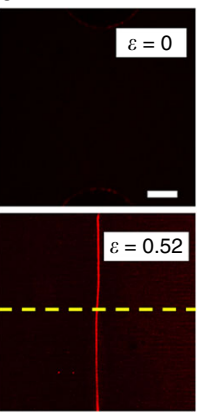
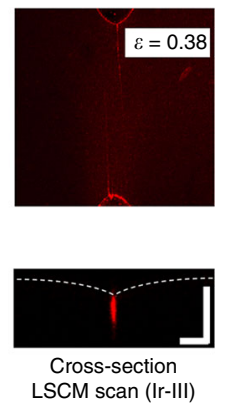

C

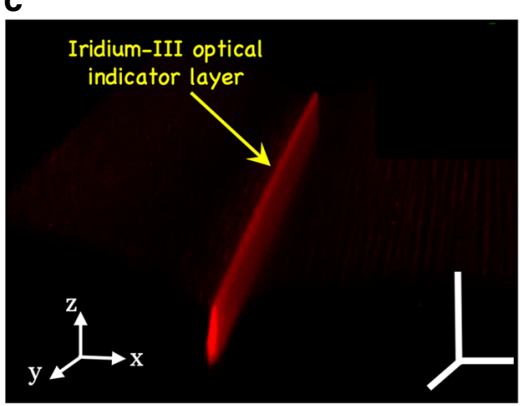

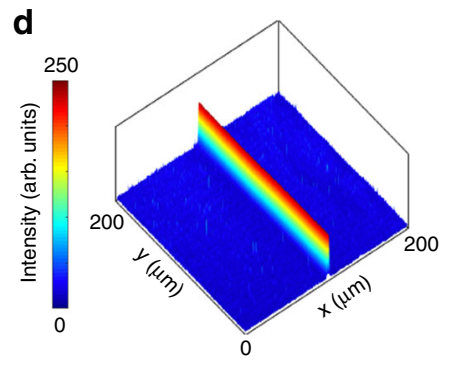
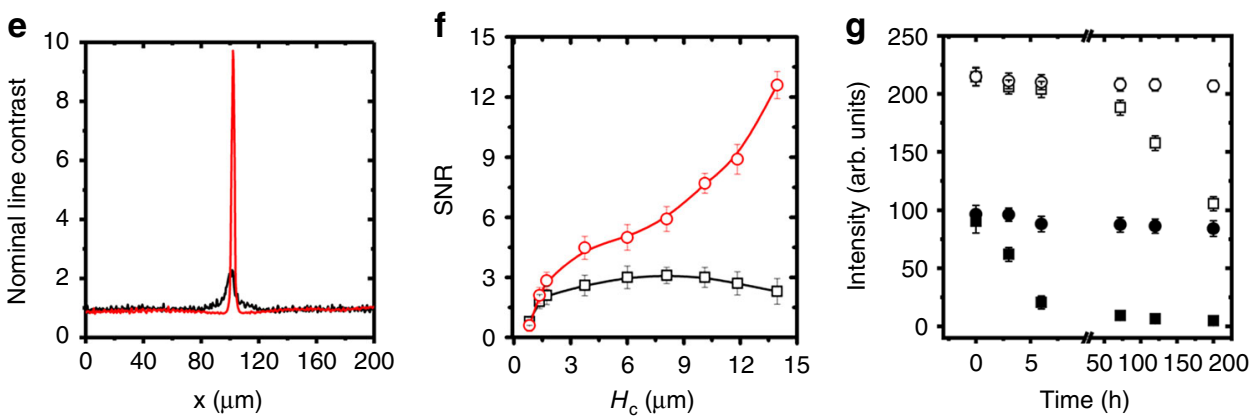

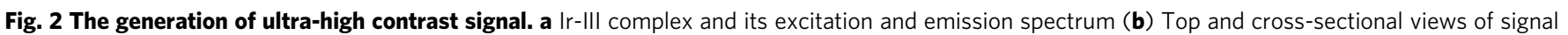

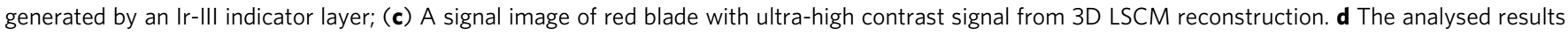

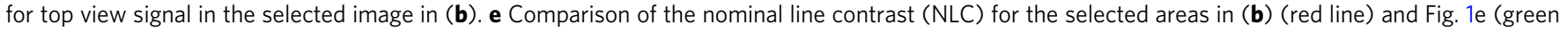
line). f The scaling of signal noise ratio (SNR) versus self-contact depth $\left(H_{c}\right)$ for fluorescein o-acrylate (FoA, black symbol) and Iridium-III (IR-III, red

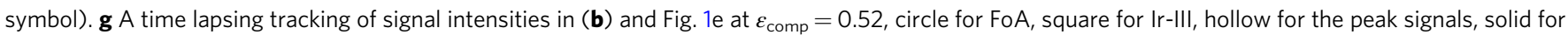
the background signals. All scale bars are $30 \mu \mathrm{m}$.

oxygen into the elastic solids. The kinetics of diffusing oxygen into the self-contact region of PDMS surface is complicated as it corresponds to the factors such as temperature, local oxygen concentration, humidity, surface porosity, chemical composition, etc, we thus defer this understanding into future study.

Numerical analysis of self-contact depth guided by surface pattern. We next perform numerical analysis with a commercial finite element simulation software to understand the mechanism of generating targeted folding. The single array of holes with varied geometrical inputs are considered to simulate the in-plane (Supplementary Movie 2) and out of plane (cross-section, Supplementary Movie 3) strain energy localization. By comparing the results for $D / \Phi=1$ (Fig. 3a) and $D / \Phi=5$ (Fig. 3b), we find that the in-plane strain concentration for $D / \Phi=1$ is stronger than that for $D / \Phi=5$. The out-of-plane (cross-section) simulation results suggest a progressing deformation with the closure of hole (initiation of $H_{\mathrm{c}}$ ), development of $H_{\mathrm{c}}$ as a folded contact with non-contact at the bottom (see Fig. $3 b$ ), then finally reaching a fully self-contact stage (creasing type, Fig. 3a).

As described above, the development of self-contact is rapid from an onsite $H_{\mathrm{c}}$ at $\varepsilon_{\mathrm{th}}$, to the $H_{\mathrm{c}}$ that can provide enough contrast. We next numerically analysis the $\varepsilon_{\text {th }}$ as a function of $D / \Phi(\Phi=40 \mu \mathrm{m})$ to study the threshold of generating optical signal guided by hole pattern. After comparing with the experimental results (Fig. 3c), the experimental results seem larger than the simulation results for $\Phi=40 \mu \mathrm{m}$, but good agreements on the overall trend are obtained for the surface, even for those surfaces patterned with different $D / \Phi$. It should be noted that we slightly over-compressed the surface to determine the closure stage for each hole under reflective optical microscope, due to the visco-elastic nature of materials. Thus, the experimental $\varepsilon_{\text {th }}$ in this paper are a little larger than the simulated ones. The simulation for surface patterned with sharp corners (diamonds, squares, triangles and hexagons) are less successful at the moment as the current simulation programme does not allow the mesh process to progress over the sharp corner, we then separate the investigation in future work.

Following to the onsite of folding, further transient development of $H_{c}$ is critical in determining the intensity of optical signal. We plot nominal self-contact depths $\left(H_{\mathrm{c}} / h\right)$ for different $D / \Phi$ $(\Phi=40 \mu \mathrm{m})$ as a function of compressive strain (Fig. $3 \mathrm{~d}$ ) to understand this geometrical development. Excellent alignments are found between the numerical outcomes and experimental results for $D / \Phi=1$ and $D / \Phi=5$, meanwhile a slight mismatch is presented for $D / \Phi=2$. A region (grey zone) with $H_{\mathrm{c}} / \mathrm{h}$ values between 0.15 and 1.2 located on a strain range of $0.39-0.59$ is outlined by analysing the experimental results where we can expect the ideal optical signals. The results from cyclic testing (Fig. 3e) indicate a good resilience on generating target folding with a desired self-contact $H_{\mathrm{c}}\left(H_{\mathrm{c}}\right.$ of $10 \mu \mathrm{m}$ for $\Phi=40 \mu \mathrm{m}$ and a $H_{\mathrm{c}}$ of $18 \mu \mathrm{m}$ for $\left.\Phi=80 \mu \mathrm{m}, D / \Phi=2\right)$, to create enough intensity of optical signal, after a short saturation period of 1-2 cycles. The marathon cyclic assessment reveals a reliable reproducibility of $H_{\mathrm{c}}$ even after 100 cycles (Supplementary Fig. 5), due to the elastic nature of multilayer. Further study on the relaxation of folding was performed by tracing $H_{c}$ over a longer time duration, to compare with the simulation results (Supplementary Fig. 6a) at a nominal compressive strain $\left(\varepsilon_{\text {comp }}\right)$ of 0.5 . The result indicates a limited relaxation in $H_{c}$, which is in the same trend with the theoretical approximation. Low hysteresis is observed during the compression/recovery cycle (Supplementary Fig. 6b). The reason could be the elastic nature of multilayer system and low surface tension ${ }^{45,46}$ after being coated by Ir-III compound. We then extend the surface design to the square lattice patterns 
a

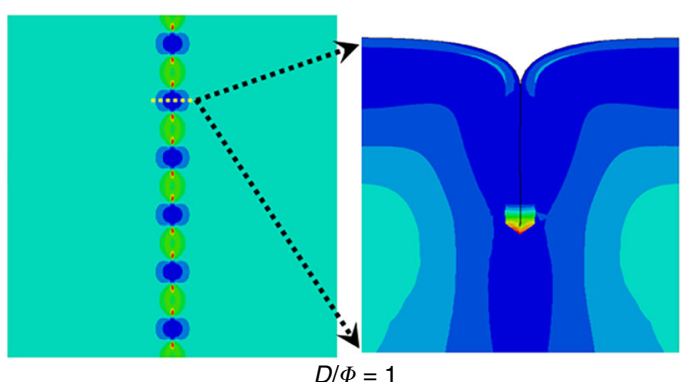

Local strain energy density $\left(\mathrm{MJ} / \mathrm{m}^{3}\right)$

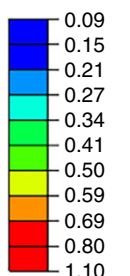

b

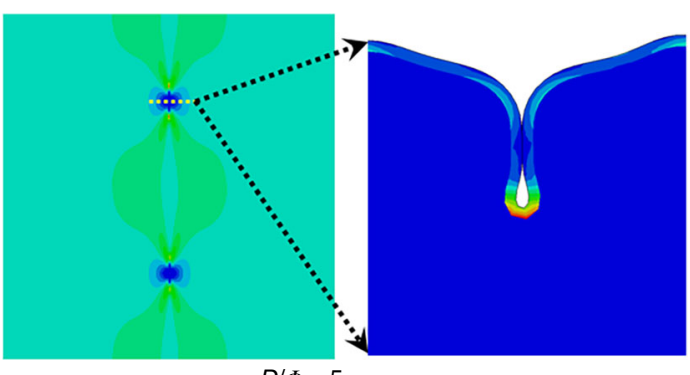

$D / \Phi=5$

e

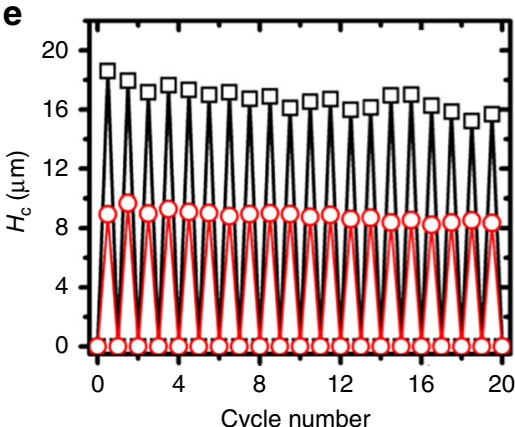

Fig. 3 Numerical analysis and reproducibility assessment. In-plane and out-of-plane strain energy analyses for the surfaces with a single micro-hole array ( $\Phi=40 \mu \mathrm{m}$ ) of (a) $D / \Phi=1$ and (b) $D / \Phi=5$, at $\varepsilon_{\text {comp }}=0.5$. c Comparison of the simulated $\varepsilon_{\text {th }}$ (threshold strain of hole closure) with experimental ones at different $D / \Phi$ for the surface with hole array ( $\square$ for $\Phi=20 \mu \mathrm{m}$, O for $\Phi=40 \mu \mathrm{m}, \Delta$ for $\Phi=60 \mu \mathrm{m}, \nabla$ for $\Phi=80 \mu \mathrm{m}$ ). d Comparison the simulation (line) and experimental (symbol) results for nominal self-contact depth $\left(H_{\mathrm{c}} / \mathrm{h}\right)$ under a progressive $\varepsilon_{\text {comp }}$ for the surface with a single micro-hole array $(\Phi=40 \mu \mathrm{m}$; black for $D / \Phi=1$, blue for $D / \Phi=2$, red for $D / \Phi=5)$. e The cyclic testing results with $\Phi=80 \mu \mathrm{m}(\square)$ and $\Phi=40 \mu \mathrm{m}(O)$, at $D / \Phi=2$.

(Supplementary Movie 4), where $W$ is defined as the distance between neighbouring lines (Supplementary Fig. 7). The results suggest that varied $\varepsilon_{\text {th }}$ at high strain can be achieved by designing $D / \Phi, W / D$ of the lattice pattern, together with more capabilities on $2 \mathrm{D}$ designs.

Demonstration of potential applications. To demonstrate the potential of developing this topo-optical sensing mechanism into device applications, an in-plane topo-optical sensor (Fig. 4a) is presented to detect large surface strains, by simply configuring the pattern parameters (shape, $D / \Phi$, etc) for the pre-placed lattice (Supplementary Movie 5). A programmable stepwise switching mechanism is encoded in this design, where a reversible line pattern could be logically switched between ' $0,0,0$ ' at $\varepsilon=0$, ' $1,0,1$ ' at $\varepsilon=0.44$ and ' $1,1,1$ ' at $\varepsilon=0.52$, with corresponding optical signals can be visualized by reflective optical microscopy and fluorescence microscopy at the same time. This concept can be further developed into dynamic 2D spy barcode products with hidden information only appearing under a dedicated stain (Fig. 4b) and an adaptive topo-optical luminescence grid (Fig. 4c), which contains a tuneable feature on the size of grid under equibiaxial compression to track the cell behaviour.

Based on this topo-optical sensing strategy, a flexible bending sensor can be developed by combining the in-plane pre-compression $\left(\varepsilon_{\text {pre-c }}\right.$, Fig. $\left.4 \mathrm{~d}\right)$ to detect out-of-the-plane bending degree. After releasing pre-stretching strain of substrate (stage I), we deploy the device on the area to detect the bending level. By observing under microscopy, the device first experiences a selective fold on the lattice patterned surface at low degree bending (stage II), then all lattice patterns are folded at high degree bending (stage III). A brief phase diagram is created to distinguish the two-stage bending sensing for the patterned surface $(\Phi=80 \mu \mathrm{m}, h=12 \mu \mathrm{m}, D / \Phi=1$ (black line) and 5 (red line), $W / \Phi=5$ ), where a clear map is obtained to determine localized curvature with the provided $\varepsilon_{\text {pre-c }}$ when the optical signal occurs.

\section{Discussion}

We have described a topo-optical sensing strategy by targeted generating folds on a micropatterned surface, with a coated optical indicator layer. The elastic multilayer shows a robust and precise optical signal by activating folds at the pre-patterned area under certain strain values. The formation of folding patterns has been studied with various geometrical inputs of the lattice patterns and the results are in a good agreement with the predictions from numerical analysis. An inherited automatic optimization on optical contrast is also introduced by oxygen quenching the Ir-III based optical indicator layer, which lead to an ultra-high contrast by significantly reducing the background noise. We anticipate this high-contrast topo-optical sensing strategy with the demonstrated conceptual devices will open new windows for future applications such as flexible/wearable electronics and bio-devices.

\section{Methods}

Fabrication of patterned multilayer elastomeric substrate. The lattice patterns were prepared through SU-8 pillar array templates photo-lithographically patterned on silicon wafer (Supplementary Fig. 8) and followed by a stamp transfer (Supplementary Fig. 9). Single-line and square (multi-line) lattice arrays of SU-8 pillars were lithographically patterned on silicon substrates to create the stamp template. Firstly, 1, 1, 1, 3, 3, 3-hexamethylsilazane (ACROS ORGANICS) was spin-coated $(30 \mathrm{~s}, 1000 \mathrm{rpm})$ onto the silicon wafer to promote adhesion. A thin layer of SU-8 (2025, Micro Chem) was then spin-coated, followed by soft baking at $95^{\circ} \mathrm{C}$ for $5 \mathrm{~min}$, before being exposed to UV light under a mask aligner (EVG 620). Post-exposure-bake was then performed $\left(65^{\circ} \mathrm{C}\right.$ for $1 \mathrm{~min}$, then ramped to $95^{\circ} \mathrm{C}$ for $5 \mathrm{~min}$ ). After being developed in an EC (ethylene lactate based) solution for $5 \mathrm{~min}$, the patterned SU-8 templates were cleaned by isopropyl alcohol and DI (de-

ionized) water. It was baked for another $15 \mathrm{~min}$ at $200^{\circ} \mathrm{C}$ before stamp transfer.

The mounting substrates were made from vinylpolysiloxane elastomer (VPS,

Elite Double 22, Zhermack) and cut into rectangular strip ( $9 \mathrm{~mm}$ wide, $30 \mathrm{~mm}$ long and $1.5 \mathrm{~mm}$ thick). The VPS strips were then mounted on mechanical vices (Supplementary Fig. 9), before being pre-stretched uniaxially to $600 \%$ strain. A thin layer $(\sim 125 \mu \mathrm{m})$ of polydimethylsiloxane (PDMS, Sylgard 184, base-to-crosslinker weight ratio $=30: 1)$ was spin-coated on the fabricated SU-8 pillars, followed by 60 min soft bake at $70^{\circ} \mathrm{C}$. An adhesion PDMS layer was then spin-coated (30:1) onto the soft-baked PDMS to bond it to the mounting VPS layer. The multilayer structures were cured at $70{ }^{\circ} \mathrm{C}$ for $8 \mathrm{~h}$. An air plasma treatment $(100 \mathrm{w}$, Henniker 
a

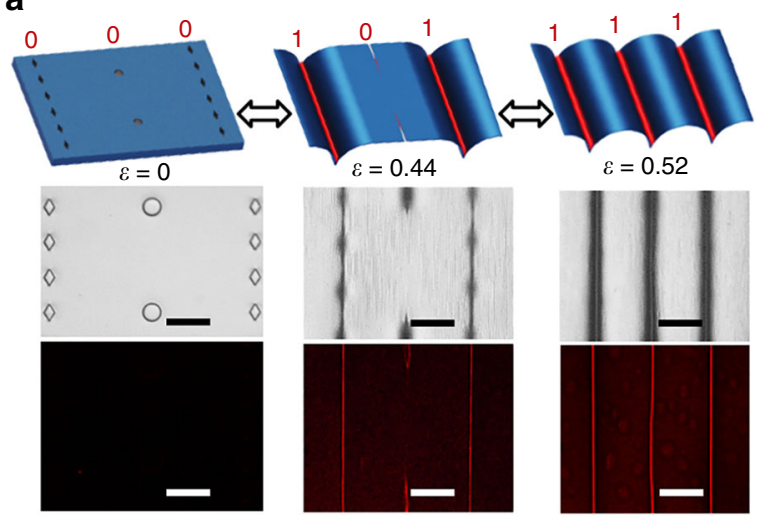

b

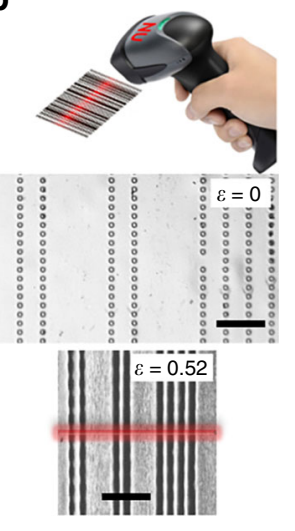

C

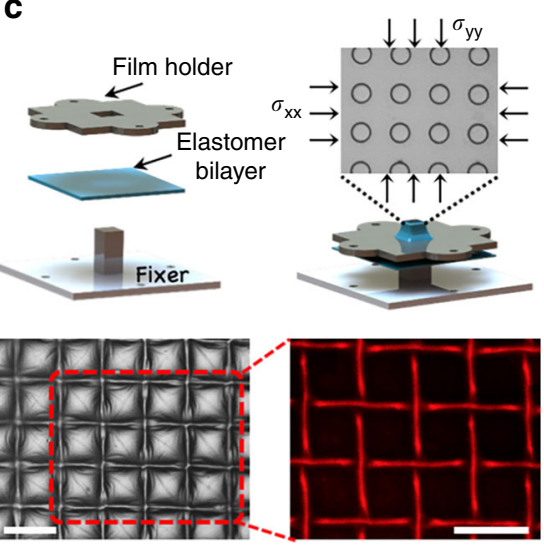

d

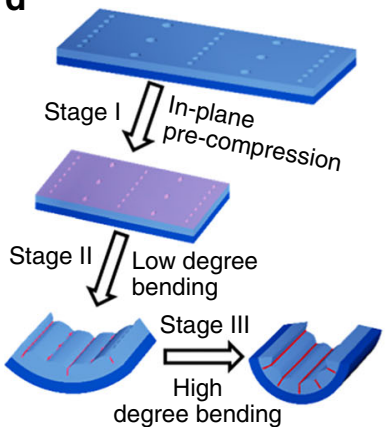

Stage I

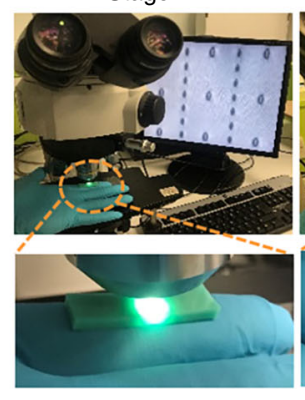

Stage II

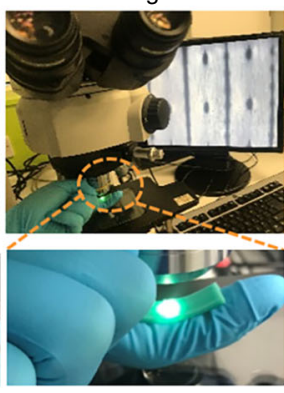

Stage III

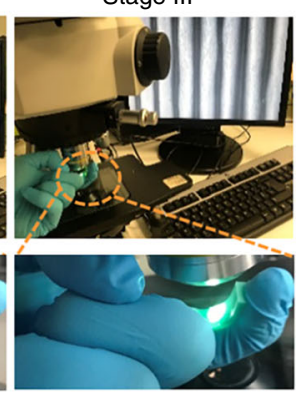

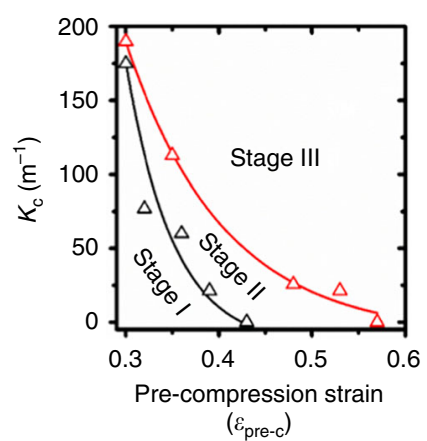

Fig. 4 Demonstration of application concepts. a An in-plane logic strain sensor with stepwise responses for large deformation sensing. $\mathbf{b}$ A 2D spy barcode design with a state of 'OFF' at $\varepsilon=0$ and an 'ON' state at $\varepsilon=0.52$. c An adaptive topo-optical grid under equi-biaxial compression. $\mathbf{d}$ A flexible tactile sensor to detect the change on local curvature with (left) schematic illustration of sensing principle, (middle) observation of conformal sensing under reflective microscopy, (right) phase diagram of the conformal tactile sensing. All scale bars are $100 \mu \mathrm{m}$.

HPT-100) of $10 s$ was applied to create a thin hard skin layer on the lattice patterned PDMS surface.

Solution casting of optical indicator layer. After the air plasma treatment, optical indicator solutions $(1.5 \mathrm{mM})$ were prepared by dissolving the dye into the absolute ethanol and chloroform. A droplet of solution containing either fluorescein Oacrylate (Sigma-Aldrich) or Iridium-III complex (synthesized in house following the published route ${ }^{47}$ ) was then casted on the surface of multilayer. The solution droplet then spread and dried at room temperature to form an optical indicator layer with a measured thickness of $\sim 600 \mathrm{~nm}$.

Characterizations. Upon releasing the pre-stretched VPS mounting substrates, the PDMS thin layer experienced uniaxial compression. Incremental deformation in a unit nominal strain of $\approx 0.004$ was applied to the sample during the compression (progressing) or tension (withdrawing/recovery) by a fixed amount at regular intervals in room temperature. A reflective optical microscope (Nikon LV-100) was used for observation under white light. $3 \mathrm{D}$ and $2 \mathrm{D}$ fluorescence imaging was performed using Nikon A1R LSCM system (LSCM). For all observations/tests, multiple measurements were performed on 7-15 selected samples (areas) to minimize the system error. For the fluorescein $\mathrm{O}$-acrylate images, the scanner selection was set to be Galvano, with laser excitation wavelength of $488 \mathrm{~nm}$ and emission wavelength of $540 \mathrm{~nm}$. For the Iridium-III images, the scanner selection was set to be Galvano, with laser excitation wavelength of $406.6 \mathrm{~nm}$ and emission wavelength of $595 \mathrm{~nm}$. The captured fluorophore images were subsequently analysed by the MATLAB to get its light intensity data and image (surf, shading interp). Standard deviations (error bars in the figures) were calculated based on the mean averaging of a group of data from 7 to 9 independent measurements on different samples.

Numerical simulation. We used the commercial simulation package-ABAQUS, to simulate surface folding on the multilayer under uniaxial compression. The incompressible neo-Hookean material model was used for all elastic materials in this analysis. Structural symmetry was assumed when the fold is simulated. The pseudo-dynamic method incorporated in ABAQUS was adopted. The geometrical inputs have been magnified by 1000 times due to the limitation of mesh size in ABAQUS. An element type CAX8H was used for mesh.

\section{Data availability}

The data that support the findings of this study are available via Northumbria Research Data Management scheme and per request from the corresponding author (B.X.).

\section{Code availability}

The numerical code developed in this work is available upon request from Dr. Xue Chen (sherry.chen@northumbria.ac.uk).

Received: 4 October 2019; Accepted: 25 February 2020; Published online: 19 March 2020

\section{References}

1. Wu, W., Wen, X. \& Wang, Z. L. Taxel-addressable matrix of vertical-nanowire piezotronic transistors for active and adaptive tactile imaging. Science 340, 952-957 (2013).

2. Pan, C. et al. High-resolution electroluminescent imaging of pressure distribution using a piezoelectric nanowire LED array. Nat. Photonics 7 , 752-758 (2013).

3. Wang, X. et al. Full dynamic-range pressure sensor matrix based on optical and electrical dual-mode sensing. Adv. Mater. 29, 1605817 (2017).

4. Wang, J. et al. Extremely stretchable electroluminescent devices with ionic conductors. Adv. Mater. 28, 4490-4496 (2016).

5. Yang, C. H., Chen, B., Zhou, J., Chen, Y. M. \& Suo, Z. Electroluminescence of giant stretchability. Adv. Mater. 28, 4480-4484 (2016).

6. Li, Y.-F. et al. Stretchable organometal-halide-perovskite quantum-dot lightemitting diodes. Adv. Mater. 31, 1807516 (2019).

7. Song, S., Song, B., Cho, C.-H., Lim, S. K. \& Jeong, S. M. Textile-fiberembedded multiluminescent devices: a new approach to soft display systems. Mater. Today https://doi.org/10.1016/j.mattod.2019.08.004 (2019).

8. Guo, Q. et al. A cephalopod-inspired mechanoluminescence material with skinlike self-healing and sensing properties. Mater. Horiz. 6, 996-1004 (2019).

9. Leung, E. M. et al. A dynamic thermoregulatory material inspired by squid skin. Nat. Commun. 10, 1947 (2019). 
10. Cao, Y. et al. Self-healing electronic skins for aquatic environments. Nat. Electron. 2, 75-82 (2019).

11. $\mathrm{Bu}, \mathrm{T}$. et al. Stretchable triboelectric-photonic smart skin for tactile and gesture sensing. Adv. Mater. 30, 1800066 (2018).

12. Sun, J. et al. Self-healable, stretchable, transparent triboelectric nanogenerators as soft power sources. ACS Nano 12, 6147-6155 (2018).

13. Mukherjee, S. \& Thilagar, P. Renaissance of organic triboluminescent. Mater. Angew. Chem. Int. Ed. 58, 7922-7932 (2019).

14. Wang, X. et al. Stretchable and transparent electroluminescent device driven by triboelectric nanogenerator. Nano Energy 58, 410-418 (2019).

15. Wong, M.-C., Chen, L., Bai, G., Huang, L.-B. \& Hao, J. Temporal and remote tuning of piezophotonic-effect-induced luminescence and color gamut via modulating magnetic field. Adv. Mater. 29, 1701945 (2017).

16. Wang, Z. in Piezotronics and Piezo-Phototronics 1-17 (Springer, Berlin, Heidelberg, 2012).

17. Yan, D. et al. Layered host-guest materials with reversible piezochromic luminescence. Angew. Chem. Int. Ed. 50, 7037-7040 (2011).

18. Zhao, H. et al. Buckling and twisting of advanced materials into morphable 3D mesostructures. Proc. Natl Acad. Sci. 116, 13239-13248 (2019).

19. Sagara, Y., Yamane, S., Mitani, M., Weder, C. \& Kato, T. Mechanoresponsive luminescent molecular assemblies: an emerging class of materials. Adv. Mater. 28, 1073-1095 (2016).

20. Zhang, J.-C., Wang, X., Marriott, G. \& Xu, C.-N. Trap-controlled mechanoluminescent materials. Prog. Mater. Sci. 103, 678-742 (2019).

21. Jeong, S. M., Song, S., Lee, S.-K. \& Ha, N. Y. Color manipulation of mechanoluminescence from stress-activated composite films. Adv. Mater. 25 6194-6200 (2013).

22. Zhao, W. et al. Highly sensitive switching of solid-state luminescence by controlling intersystem crossing. Nat. Commun. 9, 3044 (2018).

23. Bai, L. et al. Halogen-assisted piezochromic supramolecular assemblies for versatile haptic memory. J. Am. Chem. Soc. 139, 436-441 (2017).

24. Jeong, S. M., Song, S., Kim, H., Joo, K.-I. \& Takezoe, H. Mechanoluminescence color conversion by spontaneous fluorescent-dye-diffusion in elastomeric zinc sulfide composite. Adv. Funct. Mater. 26, 4848-4858 (2016).

25. Ge, D. et al. A robust smart window: reversibly switching from high transparency to angle-independent structural color display. Adv. Mater. 27, 2489-2495 (2015).

26. Zhu, Q., Van Vliet, K., Holten-Andersen, N. \& Miserez, A. A double-layer mechanochromic hydrogel with multidirectional force sensing and encryption capability. Adv. Funct. Mater. 29, 1808191 (2019).

27. Wang, D. \& Tang, B. Z. Aggregation-induced emission luminogens for activity-based sensing. Acc. Chem. Res. 52, 2559-2570 (2019).

28. v. Bünau, G. J. B. Birks: photophysics of aromatic molecules. WileyInterscience, London 1970. 704 Seiten. Preis: 210s. Berichte der Bunsengesellschaft für. Phys. Chem. 74, 1294-1295 (1970).

29. Qiu, Z. et al. Dynamic visualization of stress/strain distribution and fatigue crack propagation by an organic mechanoresponsive AIE luminogen. Adv. Mater. 30, 1803924 (2018).

30. Ji, X. et al. A functioning macroscopic "Rubik's Cube" assembled via controllable dynamic covalent interactions. Adv. Mater. 31, 1902365 (2019).

31. Liu, X. et al. In situ generation of azonia-containing polyelectrolytes for luminescent photopatterning and superbug killing. J. Am. Chem. Soc. 141, 11259-11268 (2019).

32. Lee, E. et al. Tilted pillars on wrinkled elastomers as a reversibly tunable optical window. Adv. Mater. 26, 4127-4133 (2014).

33. López Jiménez, F., Kumar, S. \& Reis, P. M. Soft color composites with tunable optical transmittance. Adv. Opt. Mater. 4, 620-626 (2016).

34. Zeng, S. et al. Bio-inspired sensitive and reversible mechanochromisms via strain-dependent cracks and folds. Nat. Commun. 7, 11802 (2016).

35. Brau, F. et al. Multiple-length-scale elastic instability mimics parametric resonance of nonlinear oscillators. Nat. Phys. 7, 56-60 (2010).

36. Xu, B., Chen, D. \& Hayward, R. C. Mechanically gated electrical switches by creasing of patterned metal/elastomer bilayer films. Adv. Mater. 26, 4381-4385 (2014).

37. Xu, B., Liu, Q., Suo, Z. \& Hayward, R. C. Reversible electrochemically triggered delamination blistering of hydrogel films on micropatterned electrodes. Adv. Funct. Mater. 26, 3218-3225 (2016).

38. De Jong, E., Wang, Y., Den Toonder, J. M. J. \& Onck, P. R. Climbing droplets driven by mechanowetting on transverse waves. Sci. Adv. 5, eaaw0914 (2019).

39. Kang, J.-H., Kim, H., Santangelo, C. D. \& Hayward, R. C. Enabling robust selffolding origami by pre-biasing vertex buckling direction. Adv. Mater. 31, 193006 (2019).

40. Silverberg, J. L. et al. Origami structures with a critical transition to bistability arising from hidden degrees of freedom. Nat. Mater. 14, 389-393 (2015)
41. Silverberg, J. L. et al. Using origami design principles to fold reprogrammable mechanical metamaterials. Science 345, 647-650 (2014).

42. Wang, D. et al. Spatially configuring wrinkle pattern and multiscale surface evolution with structural confinement. Adv. Funct. Mater. 28, 1-9 (2018).

43. Auguste, A., Jin, L., Suo, Z. \& Hayward, R. C. Post-wrinkle bifurcations in elastic bilayers with modest contrast in modulus. Extrem. Mech. Lett. 11, 30-36 (2017).

44. Zhao, R., Zhang, T., Diab, M., Gao, H. \& Kim, K.-S. The primary bilayer rugaphase diagram I: localizations in ruga evolution. Extrem. Mech. Lett. 4, 76-82 (2015).

45. Chen, D., Cai, S. Q., Suo, Z. \& Hayward, R. C. Surface energy as a barrier to creasing of elastomer films: an elastic analogy to classical nucleation. Phys. Rev. Lett. 109, 038001 (2012)

46. Yang, J., Jin, L., Hutchinson, J. \& Suo, Z. Plasticity retards the formation of creases. J. Mech. Phys. Solids 123, 305-314 (2019).

47. Turnbull, G., Williams, J. A. G. \& Kozhevnikov, V. N. Rigidly linking cyclometallated $\operatorname{Ir}($ iii) and $\mathrm{Pt}(\mathrm{ii})$ centres: an efficient approach to strongly absorbing and highly phosphorescent red emitters. Chem. Commun. 53, 2729-2732 (2017)

\section{Acknowledgements}

The work was supported by the Engineering and Physical Sciences Research Council (EPSRC) grant-EP/N007921/1 and EP/S01280X/1, and Royal Society Kan Tong Po International Fellowship 2019-KTP $\backslash$ R1 $\backslash 191012$. Dr Ben Xu and Dr Ding Wang would thank Reece Innovation for the studentship support. Professor Jie Kong thanks the financial support from Natural Science Basic Research Plan in Shaanxi Province (2018JC 008, Distinguished Young Scholar).

\section{Author contributions}

The idea was initially generated between B.X., Y.L. and V.K. B.X. and Y.L. directed the research programme and designed the experiments with C.W. C.W. carried out the major part of experiments with D.W.'s assistances on multilayer fabrication and pattern transformation. The micro-pattern template was engineered by D.W. V.K. and G.T. synthesis and characterized the Ir-III complex and diluted it into the mixed solvent for casting. B.X., X.C. and X.D. developed the analytical simulations. B.X., Y.L. J.K. V.K. and B.Z.T analyzed and interpreted the data. B.X., Y.L. J.K. and B.Z.T defined the scope together and wrote the paper with contributions from all authors.

\section{Competing interests}

The authors declare no competing interests.

\section{Additional information}

Supplementary information is available for this paper at https://doi.org/10.1038/s41467 020-15288-8.

Correspondence and requests for materials should be addressed to J.K., B.Z.T., Y.L. or B.B.X.

Peer review information Nature Communications thanks Kin Liao and the other, anonymous, reviewer(s) for their contribution to the peer review of this work. Peer reviewer reports are available.

Reprints and permission information is available at http://www.nature.com/reprints

Publisher's note Springer Nature remains neutral with regard to jurisdictional claims in published maps and institutional affiliations.

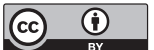

Open Access This article is licensed under a Creative Commons Attribution 4.0 International License, which permits use, sharing, adaptation, distribution and reproduction in any medium or format, as long as you give appropriate credit to the original author(s) and the source, provide a link to the Creative Commons license, and indicate if changes were made. The images or other third party material in this article are included in the article's Creative Commons license, unless indicated otherwise in a credit line to the material. If material is not included in the article's Creative Commons license and your intended use is not permitted by statutory regulation or exceeds the permitted use, you will need to obtain permission directly from the copyright holder. To view a copy of this license, visit http://creativecommons.org/ licenses/by/4.0/.

(C) The Author(s) 2020 